\title{
Two types of representation in visual memory: Evidence from the effects of stimulus contrast on image combination
}

\author{
GRAHAM J. HITCH \\ University of Lancaster, Lancaster, England \\ MARIA A. BRANDIMONTE \\ University of Trieste, Trieste, Italy \\ and \\ PETER WALKER \\ L'niversity of Central Lancashire, Preston, England
}

\begin{abstract}
An imagery task was used to explore the distinction between visual memory representations that preserve surface features and more abstract descriptions. Subjects were shown two line drawings and were then asked to combine a visual image of each drawing in order to identify a novel figure. The main experimental manipulation concerned the contrast in which each drawing was shown, which was either normal or reversed. Experiment 1 showed that image combination was more efficient when the contrasts of the two drawings were congruent with each other, but only when the imagery task was performed under conditions emphasizing short-term memory (STM). There was no effect of congruity when one of the images was generated from long-term memory (LTM). These results are consistent with the idea that the surface characteristics of a stimulus are preserved in visual STM, but a more abstract description is stored in visual LTM. In Experiment 2 we explored the influence of verbal recoding on performance of the imagery task by requiring subjects to suppress articulation. Under LTM conditions, performance of the imagery task was improved by suppression and became sensitive to contrast congruity. Under STM conditions, imagery was unaffected by suppression. Overall, these results support the distinction between surface and abstract descriptions in visual memory. However, they suggest that this distinction does not map onto that between STM and LTM in any simple way. It is suggested that short-term visual memory maintains surface descriptions and long-term visual memory preserves both surface and abstract descriptions. Verbal coding of visual stimuli appears to encourage the use of abstract visual descriptions.
\end{abstract}

Studies of visual recognition memory have suggested that there are separate short-term and long-term visual stores analogous to the short- and long-term stores in verbal memory (see, e.g., Avons \& Phillips, 1980; Phillips, 1983). Visual short-term memory (STM) is seen as a limited-capacity system that maintains a record of recently presented visual information. Long-term memory (LTM) is a more durable store for well-learned information, with no obvious limit on its storage capacity. A similar distinction appears in some theories of visual imagery, according to which visual images are maintained by using a limited-capacity short-term visual buffer (Farah, 1984; Kosslyn, 1980). Images can be generated

Part of this research was supported by a NATO collaborative research grant (CRG 911031) to G.J.H., Walter Gerbino, and P. W. We are grateful to Jonathan Schooler for comments on the interpretation of the results from Experiment 2. The experiments form part of a doctoral thesis by the second author. Requests for reprints should be addressed to G. Hitch, Department of Psychology, University of Lancaster, Lancaster LA1 4YF, England. from stored information in visual LTM or can be formed directly from perception, as a record of recent visual experience. Baddeley's (1986) model of working memory takes a similar position with its assumption that a limited-capacity store, known as the visuospatial sketchpad, is used for constructing and maintaining visual images.

A basic requirement of any theoretical account of visual memory is to specify the different kinds of information that can be represented in memory and the conditions under which these memory representations are utilized. Within the domain of verbal memory, it has frequently been suggested that different forms of representation are associated with different memory stores. For example, in verbal STM, it is believed that words are stored in terms of their acoustic or phonological properties, but in verbal LTM, they are stored in terms of their meaning (Baddeley, 1966a, 1966b). Although the issue of how different types of code map onto memory stores has proved to be both complex and controversial (see, e.g., Craik \& Lockhart, 1972), the hypothesis that an analogous distinction may apply within the domain of 
visual memory has intuitive appeal. Indeed, Humphreys and Bruce (1989, p. 201) have proposed that visual STM concerns the surface appearance of objects, whereas visual LTM involves abstract, structural descriptions of objects. Alternatively, it has been proposed that visual STM stores information about visual appearance, whereas visual LTM stores surface as well as abstract descriptions (Kosslyn, 1980). Still another view is that visual STM and visual LTM are not distinguishable in terms of the types of representation they use (Phillips, 1983).

The distinction between abstract and surface descriptions can be most readily appreciated in the context of object recognition. For example, Marr (1982) found it necessary to propose structural descriptions of objects. This type of description specifies the shape, position, and orientation of the major parts of an object relative to one another, and it is independent of the conditions under which the object is viewed on any particular occasion. In contrast, a surface description of an object specifies its appearance under a particular set of viewing conditions. Abstract visual descriptions are necessary in order for object recognition processes to cope with changes in the surface characteristics of an object under variable viewing conditions, when parameters such as illumination, distance, and orientation alter.

There is some psychological evidence supporting the distinction between abstract (object-level) and surface (view-specific) representations of visual stimuli (Bartram, 1976; Cooper, Schacter, Ballesteros, \& Moore, 1992; Ellis, Allport, Humphreys, \& Collis, 1989; Humphreys \& Riddoch, 1987; Humphreys, Riddoch, \& Quinlan, 1988; Marshall \& Walker, 1987). This evidence comes largely from studies of priming effects and recognition memory. In the present paper, we report our attempt to obtain independent support for the abstract/ surface distinction by studying visual imagery. At the same time, we planned to test the predictions of the hypothesis that visual STM is specialized for storing surface descriptions, whereas visual LTM is specialized for storing abstract descriptions (Humphreys \& Bruce, 1989). Research on visual STM has suggested that it can preserve surface features such as size, shape, and color (Larsen \& Bundesen, 1978; Milliken \& Jolicoeur, 1992; Stefurak \& Boynton, 1986), and studies of visual imagery suggest that images also typically possess surface features (Finke \& Schmidt, 1977; Kosslyn, 1980; Shephard \& Metzler, 1971). Taken together, therefore, studies of visual memory and imagery agree in suggesting that representations in visual STM specify the surface appearance of objects. However, it appears that there has not yet been a systematic attempt to explore the possibility of differences between the types of representation stored in visual STM and visual LTM.

The logic of the present experimentation involved a methodology used in previous studies (Brandimonte, Hitch, \& Bishop, 1992a, 1992c), in which the ability to manipulate an image of a remembered visual stimulus is taken as an index of memory for its physical appearance. Subjects were required to combine a mental image of a line drawing with an image of a second figure. Successful combination revealed a new form, which the subjects had to identify (see Figure 1). From the subject's point of view, the combination task involves superimposing a remembered image and a current image in the "mind's eye" in order to "see" the new form. In different conditions of the experiment, the remembered image was either (1) present in visual STM due to a recent presentation of the stimulus, or (2) generated from visual LTM following an earlier learning phase. Image manipulation was considered essential in order to perform the combination task, since the stimuli were devised in such a way that the new form could not be guessed from either part in isolation.

\section{EXPERIMENT 1}

In Experiment 1 we investigated whether information about stimulus contrast is represented in visual STM and visual LTM. Contrast information was chosen, since it is a good example of the kind of information expected to be encoded in a surface description of a visual stimulus, but not in a more abstract, structural description. In the incongruent contrast condition, one of the two line drawings presented to subjects for mental combination was shown in normal contrast (black lines on a white background), and the other was shown in reversed contrast (white lines on a black background). In the congruent contrast condition, both stimuli were shown in the same contrast (either normal or reversed).

If visual memory preserves information about stimulus contrast, image combination should be impaired when the contrasts of the two stimuli are incongruent. Subjects would presumably be unable to combine images with different contrasts by merely superimposing them in the "mind's eye." Additional processes would be required, such as transforming one of the images into the same contrast, and these extra operations would be likely to result in increased error. If, on the other hand, visual memory stores abstract descriptions, the surface characteristics of images will take on prototypical or default values, and there is therefore no reason to expect an effect of contrast congruity.

The hypothesis that visual STM contains surface descriptions and that visual LTM specializes in preserving abstract visual descriptions predicts that image combination will be impaired by contrast incongruity under STM conditions, but not under LTM conditions. An appropriate test of each of these predictions is a planned comparison of performance on the imagery task for pairs of stimuli presented in either congruent or incongruent contrasts. Differences in the overall levels of performance in STM and LTM conditions are not themselves of interest here, since the task parameters used in these two conditions were arbitrary, being chosen in order to give similar performance levels in STM and LTM. 
$\mathbf{A}$<smiles>[120I-]</smiles><smiles>C1CC[C@@H]2CC[C@@H]2C1</smiles><smiles>c1ccc2ccccc2c1</smiles><smiles>CCC[O]</smiles><smiles>[C]1CCCC1</smiles>

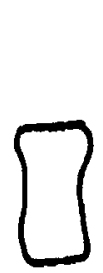

B
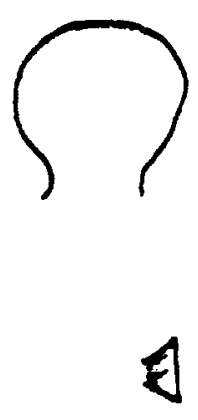

1
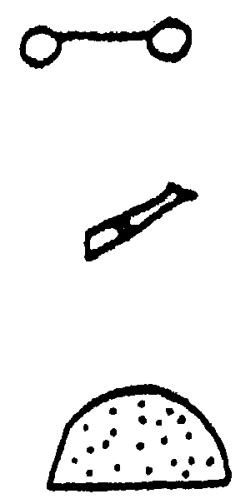

Figure 1. Examples of the image combination task. Each pair of stimuli (columns $A$ and $B$ ) is shown separately and is then combined, using mental imagery, to reveal a new form. (Stimuli were presented on rectangular cards, which indicated the correct alignment for superimposing their images.)

\section{Method}

Subjects. Eighty volunteers between the ages of 18 and 30 were recruited from the Trieste University library. They were randomly assigned to each of the four conditions obtained by crossing the factors of memory condition (STM/LTM) and contrast agreement (congruent/incongruent), subject to the constraint that there were equal numbers of males and females in each condition.

Materials. Two sets of stimuli were prepared on cards measuring $17 \times 22 \mathrm{~cm}$. Each set consisted of six pairs of figures. When superimposed, each pair of figures formed a novel composite figure that was easily named. One set consisted of simple line drawings (black lines on a white background), as those used in previous experiments (see Figure 1 and Brandimonte et al., 1992b). The other set consisted of the same six pairs of figures, with the difference that they were drawn as white lines on a black background (see Figure 2). Three further pairs of figures were drawn on transparencies in normal contrast for use in training.
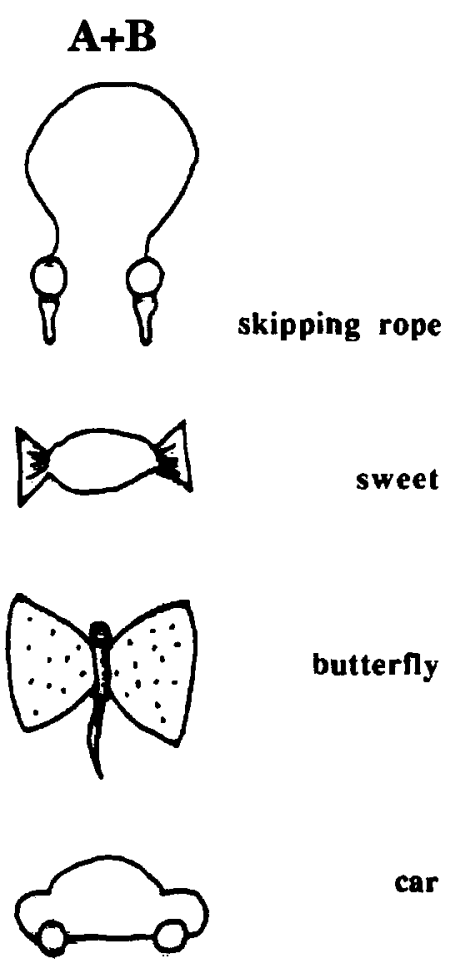

car

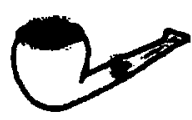

pipe

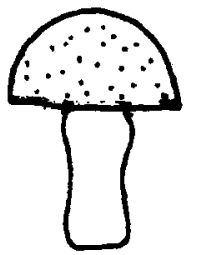

mushroom
Procedure. The subjects were tested individually. In the training phase, the first pattern of the first practice pair was presented for $2 \mathrm{sec}$, and was followed immediately by presentation of the second pattern of the pair. At this point, the experimenter superimposed the two transparencies by sliding one above the other to provide a physical demonstration of how, when precisely superimposed, the two patterns formed a new figure. The subject was then requested to name the new composite picture resulting from the combination. This training procedure was repeated three times, each time with a different pair of stimuli.

In the STM condition, each subject was shown the card containing the first member of the first pair of experimental figures for $2 \mathrm{sec}$. At the end of this period, the card was removed from view and was replaced by the card showing the second member of the pair, again for $2 \mathrm{sec}$. Immediately after presentation of the second card, the subject was requested to combine the two figures by using visual imagery and to name the new figure resulting from 

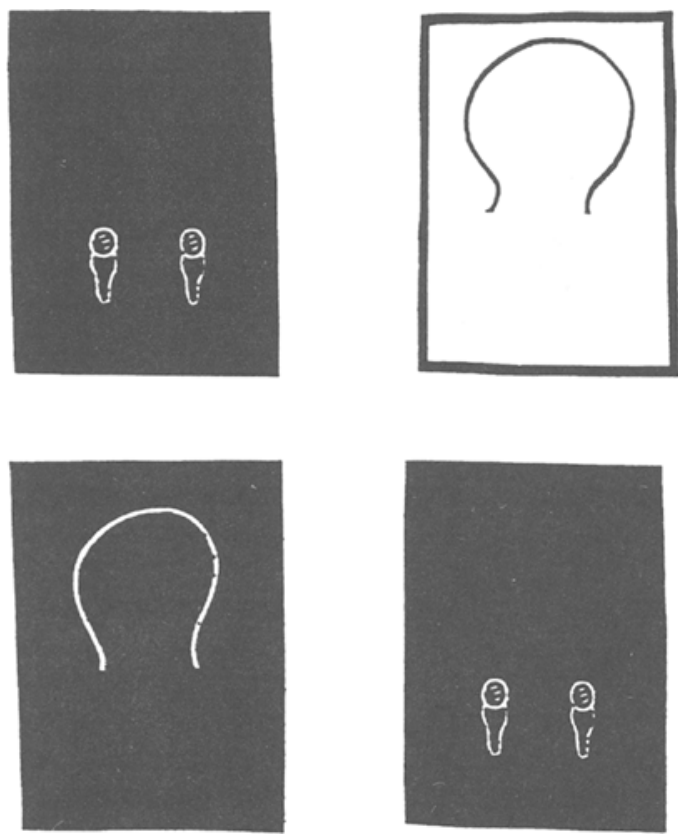

Figure 2. Examples of pairs of stimuli used in the image combination task, shown in congruent and incongruent contrasts.

this operation. This procedure was repeated for all six pairs of experimental figures. In the LTM condition, the subjects were required to learn the first members of all six pairs to the criterion that they were confident that they could remember them in the order in which they had been learned. To achieve this, the series of six figures was shown three times at a rate of $5 \mathrm{sec} /$ item (this procedure has been used successfully in previous studies; see Brandimonte et al., 1992a, 1992c). Immediately after learning, the subjects were asked to generate an image of the first figure of the learned series. When they indicated that they were ready, they were shown the second member of the pair for $2 \mathrm{sec}$ and were asked to combine the two figures mentally to discover the new form. Once they had named the resulting composite figure, the procedure was repeated until all six figure combinations had been attempted.

In each memory condition, half the subjects saw the two figures of each pair in the same contrast (either black lines on a white background or white lines on a black background), while half saw them in the opposite contrast (either the first pattern black on white and the second white on black, or vice versa). The presentation order of the different contrast conditions was counterbalanced across subjects, so that half undertook them in one order and half in the reverse order.

\section{Results}

A response to the combination task was counted as correct if it was included among, or was an obvious synonym of, the names given by a separate group of control subjects to drawings of the combined stimuli (as reported in Brandimonte et al., 1992b). Table 1 and Figure 3 show the mean numbers of correct responses. Planned comparisons showed that, in the STM condition, there was a large and significant impairment in performance of the imagery task when the two stimuli were presented in incongruent contrasts $\left[F(1,76)=9.47, M S_{\mathrm{e}}=1.53, p<\right.$
.01]. However, in the LTM condition, there was only a small, nonsignificant impairment associated with contrast incongruity $[F(1,76)=1.33]$.

\section{Discussion}

The results support the contention that memory representations for visual stimuli can differ in the extent to which they preserve information about stimulus contrast. Thus, the absence of an effect of contrast congruity in the LTM condition suggests that performance of the imagery task was based on abstract visual descriptions that did not contain information about stimulus contrast. Abstract descriptions must nevertheless contain shape information, since performance on the image combination task was clearly above chance. In the STM condition, on the other hand, the adverse effect of presenting stimuli in incongruent contrasts supports the view that visual STM preserves information about stimulus contrast. However, it is interesting that the subjects were able to get some items correct in the STM condition, even when pairs of stimuli were presented in incongruent contrasts. This suggests that contrast information can be transformed in visual STM, though at some cost, in order to combine images. Alternatively, subjects may have access to abstract as well as surface descriptions in the visual STM condition.

Overall, the pattern of results is consistent with a distinction between surface and abstract descriptions in visual memory. The results are also consistent with the suggestion that visual STM and visual LTM are differentially associated with these two types of representation (Humphreys \& Bruce, 1989). Notice, however, that the data do not rule out the possibility that abstract visual descriptions make some contribution to performance under STM conditions, as argued previously. Nor do they rule out the possibility that further investigation might yield evidence for the preservation of surface descriptions in visual LTM. Indeed, studies of recognition memory have demonstrated memory for surface infor-

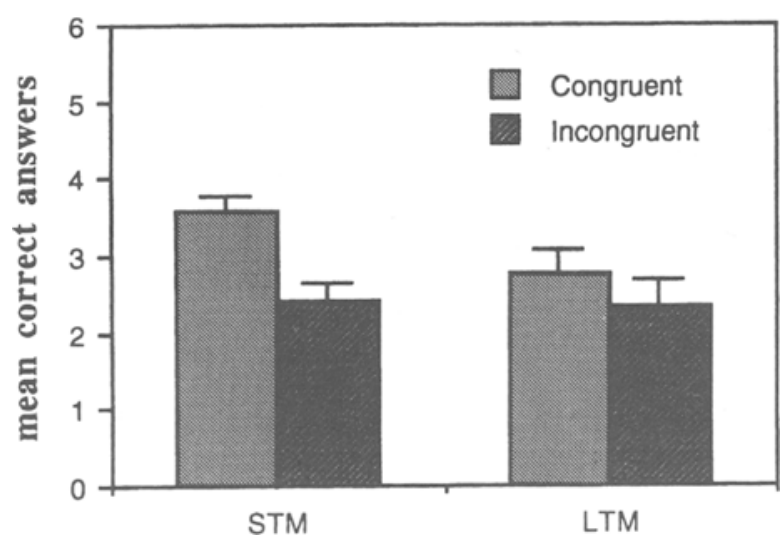

Figure 3. Results of Experiment 1. Number of correct responses (maximum $=6$ ) as a function of experimental condition (STM, shortterm memory; LTM, long-term memory). 
Table 1

Mean Number of Correct Responses on the Image Combination Task in Experiment 1 (Maximum $=6$ )

\begin{tabular}{|c|c|c|}
\hline \multirow[b]{2}{*}{ Memory Condition } & \multicolumn{2}{|c|}{ Contrast Agreement } \\
\hline & Congruent & Incongruent \\
\hline STM & 3.60 & 240 \\
\hline LTM & 275 & 2.35 \\
\hline
\end{tabular}

Note-STM, short-term memory; LTM, long-term memory.

mation over relatively long intervals (e.g., Marks, 1991). It may be, therefore, that some aspect of the present imagery task discouraged the use of surface descriptions in the LTM condition. One obvious possibility is verbal coding. The stimuli used in the image combination task were ones that subjects tend to label (Brandimonte et al., $1992 \mathrm{~b}$ ), and it is known that performance in visual memory tasks can be impaired by the presentation of verbal information (Carmichael, Hogan, \& Walter, 1932; Schooler \& Engsteler-Schooler, 1990). The results of Experiment 1 may therefore be specific to stimuli that have undergone verbal recoding, rather than a reflection of the general characteristics of performance in visual LTM and STM conditions. Experiment 2 was an attempt to investigate this issue.

\section{EXPERIMENT 2}

The work of Paivio (1971) and others has suggested that although the visual and verbal coding systems are independent, they are also closely interconnected, so it is possible to get from one to the other very easily. The effect of verbal recoding on visual imagery has recently been explored by using articulatory suppression, an interfering task that has been shown to prevent subjects from verbally recoding visual stimuli (see Baddeley, 1986). Brandimonte et al. (1992a) demonstrated that performing suppression while learning the appearance of a readily nameable visual stimulus enhanced the ability to generate an accurate mental image of the stimulus. Brandimonte et al. (1992c) extended these findings by showing that the beneficial effect of articulatory suppression was restricted to stimuli that are easy to name. They also showed that providing a plausible verbal label for a stimulus that is difficult to name interferes with the ability to generate an image of the stimulus.

The above studies agree in suggesting that verbal recoding can disrupt memory performance relating to the visual characteristics of a stimulus. However, it is interesting to note that the subjects were typically tested on relatively well-learned material after some delay. In an experiment in which subjects were required to manipulate an image of a nameable stimulus immediately after a single presentation, Brandimonte et al. (1992a, Experiment 2) found no effect of articulatory suppression. This suggests that verbal recoding influences visual LTM to a much greater extent than visual STM. The idea that visual and verbal codes interact in LTM is consistent with the claim that LTM contains modality-independent conceptual representations (Nelson, Reed, \& Walling, 1976), which are closely connected to modality-specific representations. The idea that there is little interaction between visual and verbal codes in STM is consistent with the working-memory model (Baddeley, 1986), which proposes that the visuospatial sketchpad and the phonological short-term store are functionally separate subsystems that are not directly connected.

In Experiment 2 we used articulatory suppression to investigate the influence of verbal recoding on performance of the image combination task. Expectations for the LTM condition were that suppression would enhance image combination, replicating Brandimonte et al. (1992a, 1992c). Furthermore, if visual LTM is restricted to using abstract visual descriptions, there should continue to be no effect of contrast congruity, even under articulatory suppression. However, if verbal recoding merely introduces a bias toward using abstract descriptions in visual LTM, blocking verbal recoding will encourage the use of surface descriptions. On this view, articulatory suppression should not only improve image combination in the LTM condition, but should also entail that performance becomes sensitive to contrast congruity.

Predictions for the effect of suppression in the visual STM condition were more straightforward. If it is assumed that visual STM is functionally independent of verbal STM, then blocking verbal recoding should have no effect on the imagery task and performance should be more difficult when the figures are seen in incongruent contrasts. Following the rationale used in Experiment 1, we tested these predictions with planned comparisons.

\section{Method}

Subjects. Recruitment for the one hundred twenty-eight subjects was the same as that for Experiment 1 . We used a betweensubjects $2 \times 2 \times 2$ design with memory conditions (STM/LTM), contrast agreement (congruent/incongruent), and articulatory suppression (present/absent) as factors. The subjects were randomly assigned to the eight experimental conditions with the constraint that there were equal numbers of males and females in each.

Materials and Procedure. The materials and procedure were the same as those in Experiment 1, with the exception that in the articulatory suppression conditions, the subjects repeated an irrelevant sound ("la-la-la") while encoding the first picture of each pair. More specifically, the subjects suppressed articulation throughout the initial learning phase in the LTM conditions. In the STM conditions, they suppressed during the $2 \mathrm{sec}$ of each trial in which they were shown the first figure of each pair. Suppression was not required during performance of the imagery task.

\section{Results}

Performance was scored by using the criteria used in Experiment 1. Table 2 and Figure 4 show the mean number of correct responses in the image combination task in each condition. First, it is clear that articulatory suppression had no effect on performance in the STM condition $\left[F(1,120)<1, M S_{\mathrm{e}}=1.36\right]$, but suppression significantly enhanced performance in the LTM condition $[F(1,120)=5.60, p<.025]$. Second, in the absence of articulatory suppression, the effects of contrast con- 

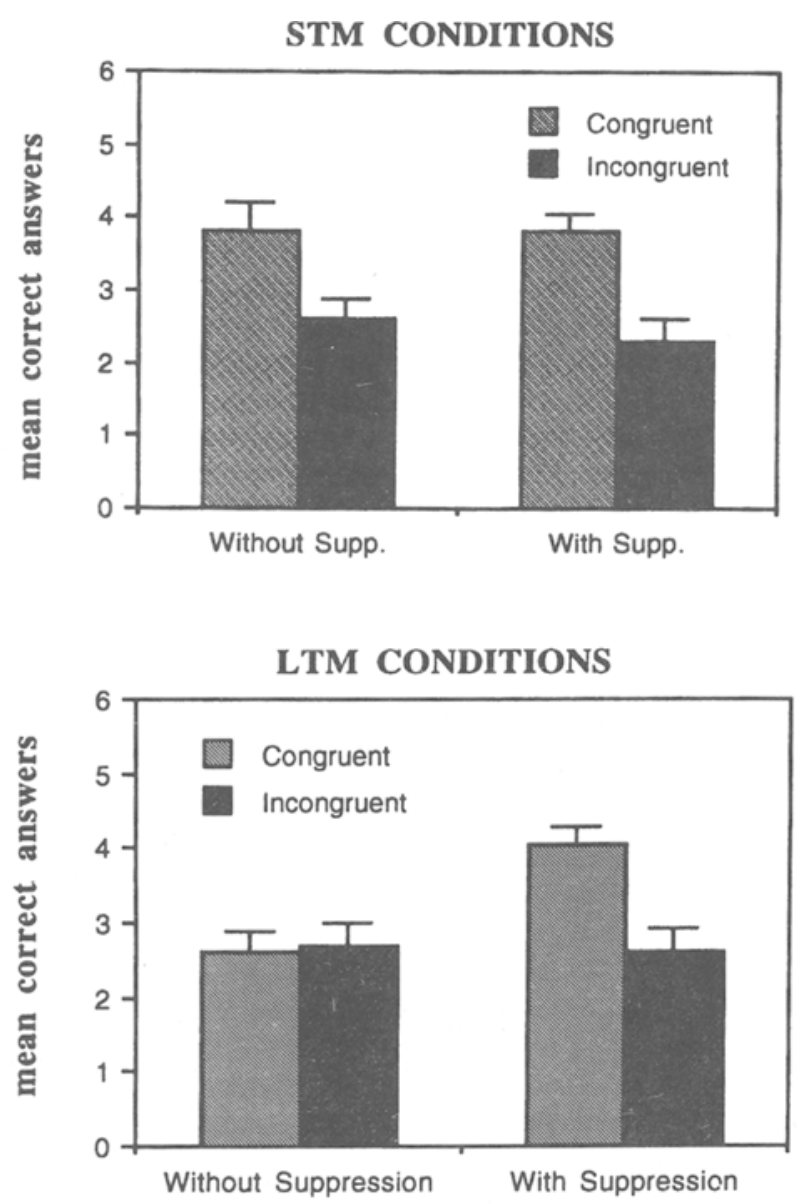

Figure 4. Results of Experiment 2. Number of correct responses (maximum $=6$ ) in the short-term memory (STM) conditions and the long-term memory (LTM) conditions.

gruity replicated the pattern observed in Experiment 1. Thus, in the STM condition, imagery was impaired when the stimuli were presented in incongruent contrasts $[F(1,120)=16.66, p<.001]$, but in the LTM condition there was no effect of contrast $(F<1)$. Finally, the pattern of results was quite different when the subjects performed articulatory suppression. In particular, contrast incongruity impaired image combination in both

Table 2

Mean Number of Correct Responses on the Image Combination Task in Experiment 2 (Maximum $=6$ )

\begin{tabular}{|c|c|c|}
\hline \multirow[b]{2}{*}{ Memory Condition } & \multicolumn{2}{|c|}{ Contrast Agreement } \\
\hline & Congruent & Incongruent \\
\hline \multicolumn{3}{|c|}{ Control (No Suppression) } \\
\hline STM & 3.81 & 2.62 \\
\hline LTM & 2.62 & 2.68 \\
\hline \multicolumn{3}{|c|}{ Articulatory Suppression } \\
\hline STM & 3.81 & 2.31 \\
\hline LTM & 4.06 & 2.68 \\
\hline
\end{tabular}

Note-STM, short-term memory; LTM, long-term memory. the STM and the LTM conditions $[F(1,120)=26.47$, $p<.001$, and $F(1,120)=24.39, p<.001$, respectively]

\section{Discussion}

The pattern of performance in the absence of articulatory suppression is consistent with that shown in Experiment 1, but the difference between STM and LTM is even more marked. Thus, the significant advantage of presenting stimuli in congruent contrasts in the STM condition compares with a nonsignificant difference in the reverse direction in the LTM condition. This aspect of the results provides further evidence for the claim that imagery performance in the STM condition involved the use of representations that preserved surface information, whereas performance in the LTM condition involved abstract visual descriptions. However, the effects of articulatory suppression indicate that this pattern of results reflects the influence of verbal recoding. Suppression enhanced image combination when one of the images was generated from visual LTM, and a clear effect of contrast congruity emerged, indicating that the generated image was based on a surface description. This result argues strongly against the idea that visual LTM preserves only abstract descriptions (Humphreys $\&$ Bruce, 1989). Overall, the present results favor the view that visual LTM preserves surface and abstract descriptions, and they suggest that verbal recoding is associated with a bias toward using abstract visual descriptions. Notice that the effect of verbal coding cannot readily be explained by appealing to the use of nonvisual representations, such as conceptual or semantic codes. By their nature, such codes would not contain the shape information necessary to support performance in the image combination task.

The absence of any effect of suppression in the STM condition is consistent with the idea that visual STM is functionally independent of verbal STM, and the marked contrast in the importance of suppression in the STM and LTM conditions supports the contention that the interaction between the visual and verbal coding systems arises chiefly in relation to LTM.

\section{GENERAL DISCUSSION}

The present experiments illustrate how simple visual imagery tasks can be used to address basic questions about the nature of representations in visual memory. In particular, they support a distinction between representations that preserve information about stimulus contrast and those that, although preserving shape information, contain no information about contrast. More generally, the results show that imagery tasks can provide valuable converging evidence for the distinction between surface and abstract visual representations, which has, up to now, been based largely on the study of priming effects and neuropsychological data (Bartram, 1976; Cooper et al., 1992; Ellis et al., 1989; Humphreys \& Riddoch, 1987; Humphreys et al., 1988). There are, however, two 
important qualifications. First, given the preliminary nature of the present investigation, the findings are necessarily restricted in their scope. Two important goals for future study are (1) to extend the investigation to include the representation of other surface features besides contrast, and (2) to attack more directly the nature of abstract visual descriptions. Second, it might be argued that the present evidence can be explained in terms of differences in the amount of information that is represented in visual memory, rather than a distinction between separate types of representation. However, such an account makes it difficult to see why less information should be stored when verbalization is allowed, and it fails to capture the possibility that the absence of information about contrast and other surface characteristics of a stimulus may be advantageous in some circumstances (e.g., perceptual constancy). Although these qualifications suggest the need for caution in generalization and interpretation, it is important to note also that the overall pattern of results was closely in accordance with predictions based on previous work.

Evidence about the conditions under which surface and abstract visual representations are used is just starting to accumulate, and currently very little is known. The present data suggest that use of surface representations is favored in tasks that tap visual STM, whereas the use of abstract representations is encouraged by verbal recoding in tasks that tap visual LTM. It could be argued that since performance with incongruent stimuli in the STM and LTM conditions was virtually identical, the same processes mediated performance. However, as we have already pointed out, comparing the overall levels of performance between the STM and LTM conditions is not very meaningful, since task parameters (e.g., exposure durations) were chosen in order to make these levels similar. Nevertheless, it is important to consider the implications of the finding that performance in the STM condition with incongruent stimuli was clearly above chance level. If the subjects were able to reverse the contrast of either stimulus before combining them, then performance in this condition could be based on surface representations. However, an alternative possibility is that performance was mediated by abstract representations. Indeed, the abstract representations identified with LTM may have supported performance in this condition. If this were the case, however, the pattern of STM performance ought to have shown the same sensitivity to articulatory suppression that LTM did, which was not the case. This leaves the possibility that there may be a type of abstract representation that is unique to STM and that is insensitive to the effects of suppression. Although such an interpretation cannot be ruled out at this stage, it provides a more complex account than the simpler assumption that subjects are able to reverse the contrast of a visual image in STM. Whichever account is preferred, the distinction between visual STM and visual LTM is reinforced by their differential sensitivity to articulatory suppression.
Evidence from other sources has suggested that surface representations take less time to generate, are less durable, and are more sensitive to interference than abstract (object-level) representations (Ellis et al.,1989). It has also been suggested that abstract descriptions are used in implicit memory tasks, whereas surface descriptions are used in tests of explicit (episodic) memory (Cooper et al., 1992). The present results therefore emphasize the need to broaden and intensify the investigation of the properties of abstract and surface descriptions in visual memory. In this context, the claim that both abstract and surface information are preserved in visual LTM can be seen to be consistent with our ability to recognize a specific object among other objects with similar visual structure but different physical appearance (e.g., one's own car in a parking lot). This claim would also suggest that generating an image from visual LTM is similar to other forms of remembering, insofar as schematic knowledge as well as information about literal detail are involved (Bartlett, 1932). A much more surprising aspect of the present findings is the evidence that verbal recoding can influence the type of visual in formation used to generate an image from LTM, by encouraging the use of abstract descriptions.

Figure 5 shows a simplistic model, which can account for the evidence reported here. It is intended to illustrate how the results can be explained, since there are many alternative ways of interpreting them. The model is essentially a combination of recent accounts of visual memory and visual imagery (Farah, 1984; Kosslyn, 1980), with views about the verbal memory system. Importantly, it includes the ideas, taken from the workingmemory model of Baddeley (1986), that there are independent visual and verbal short-term stores that are not directly interconnected, and that verbally recoding visual stimuli involves subvocal articulation. Distinctive aspects of the model are its specification of the kinds of information preserved by different parts of the visual memory system and the idea that the visual and verbal

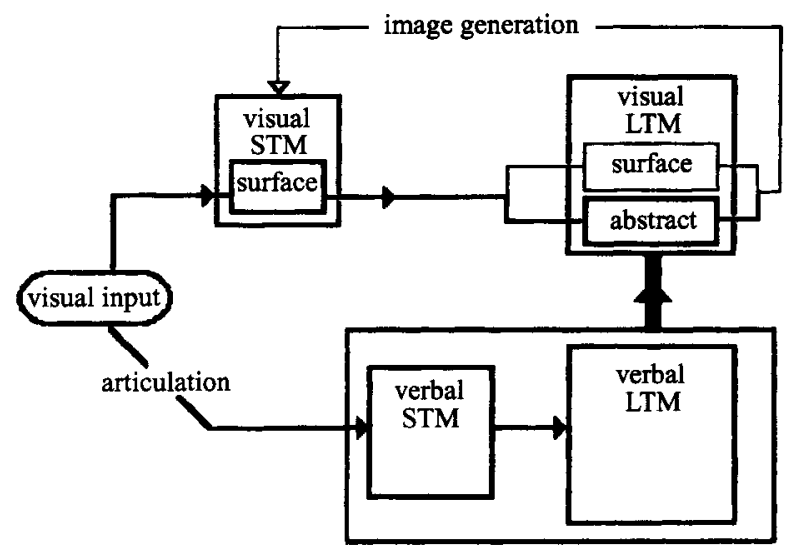

Figure 5. A simplified model of the way pictorial stimuli access the visual and verbal memory systems. The model accommodates the present results. 
memory systems interact in LTM and not STM. Following Kosslyn and Farah, visual STM is seen as a buffer for recent visual inputs as well as for images generated from LTM. Visual STM is also assumed to be the workspace where imagery operations are performed and is assumed to store descriptions of the surface appearance of visual stimuli, whereas visual LTM preserves both surface and abstract descriptions. To capture the fact that visual inputs can also be given verbal descriptions, the model includes a mechanism whereby such descriptions are generated in verbal STM and stored more permanently in verbal LTM. As in the working-memory model, visual and verbal STM are not directly connected, and the effect of articulatory suppression is to stop information about a visual stimulus from being recoded in verbal STM. The present model assumes that visual and verbal LTM are closely connected, so that the availability of a description of a stimulus in verbal LTM encourages the use of abstract descriptions in visual LTM. In this way, the model gives a straightforward account of the effect of articulatory suppression on imagery, since preventing verbal recoding removes the bias toward using abstract descriptions. The proposed account therefore retains the strengths of current theoretical approaches to visual and verbal memory and in addition specifies how the visual and verbal systems may be interconnected.

\section{REFERENCES}

Avons, S. E., \& Phillips, W. A. (1990). Visualization and memorization as a function of display time and poststimulus processing time. Journal of Experimental Psychology: Human Learning \& Memory, 6, 407-420.

BADDELEY, A. D. (1966a). The influence of acoustic and semantic similarity on long-term memory for word sequences. Quarterly Journal of Experimental Psychology, 18, 302-309.

BADDELEY, A. D. (1966b). Short-term memory for word sequences as a function of acoustic, semantic and formal similarity. Quarterly Journal of Experimental Psychology, 18, 362-365.

BADDELEY, A. D. (1986). Working memory. Oxford: Oxford University Press, Clarendon Press.

BARTLETT, F. C. (1932). Remembering: A study in experimental and social psychology. Cambridge: Cambridge University Press.

BARTRAM, D. J. (1976). Levels of coding in picture-picture comparison tasks. Memory \& Cognition, 4, 593-602.

Brandimonte, M. A., Hitch, G. J., \& Bishop, D. V. M. (1992a). Influence of short-term memory codes on visual image processing: Evidence from image transformation tasks. Journal of Experimental Psychology: Learning, Memory, \& Cognition, 18, 157-165.

Brandimonte, M. A., Hitch, G. J., \& Bishop, D. V. M. (1992b). Manipulation of visual mental images in children and adults. Journal of Experimental Child Psychology, 53, 300-312.

Brandimonte, M. A., Hitch, G. J., \& Bishop, D. V. M. (1992c). Verbal recoding of visual stimuli impairs mental image transformations. Memory \& Cognition, 20, 449-455.
Carmichael, L. C., Hogan, H. P., \& Walter, A. A. (1932). An experimental study of the effect of language on the reproduction of visually perceived form. Journal of Experimental Psychology, 15, 73-86.

Cooper, L. A., Schacter, D. L., Ballesteros, S, \& Moore, C. (1992). Priming and recognition of transformed three-dimensional objects: Effects of size and reflection. Journal of Experimental Psychology: Learning, Memory, \& Cognition, 18, 43-57.

Craik, F. I. M., \& LockHaRT, R. S. (1972). Levels of processing: A framework for memory research. Journal of Verbal Learning \& Verbal Behavior, 11, 671-684.

Ellis, R., Allport, D. A., Humphreys, G. W., \& Collis, J. (1989). Varieties of object constancy. Quarterly Journal of Experimental Psychology, 41A, 775-796.

FARAH, M. (1984). The neurological basis of mental imagery: A componential analysis. Cognition, 18, 241-269.

FINKE, R. A., \& SCHMIDT, M. J. (1977). Orientation-specific color after-effects following imagination. Journal of Experimental Psychology: Human Perception \& Performance, 3, 599-606.

Humphreys, G. W., \& BRUCE, V. (1989). Visual cognition. Computational, experimental and neuropsychological perspectives. Hove, U.K.: Erlbaum.

HUMPHREYS, G. W., \& RIDDOCH, J. M. (1987). The fractionation of visual agnosia. In G. W. Humphreys \& J. M. Riddoch (Eds.), Visual object processing: A cognitive neuropsychological approach (pp. 281306). Hove, U.K.: Erlbaum.

HumphreYs, G. W., RidDoch, J. M., \& QuinLAN, P. T. (1988). Cascade processes in picture naming. Cognitive Neuropsychology, 5, 67-104.

Kosslyn, S. M. (1980). Image and mind. Cambridge, MA: Harvard University Press.

LARSEN, A., \& BUNDESEN, C. (1978). Size scaling in visual pattern recognition. Journal of Experimental Psychology: Human Perception \& Performance, 4, 1-20.

MARKS, W. (1991). Effects of encoding the perceptual features of pictures on memory. Journal of Experimental Psychology: Learning, Memory, \& Cognition, 17, 566-577.

MARR, D. (1982). Vision San Francisco: W. H. Freeman.

MARShall, E. L., \& WALKER, P. (1987). Visual memory for pictorial stimuli in a serial choice reaction time task. British Journal of Psychology, 78, 213-231.

Milliken, B., \& Jolicoeur, P. (1992). Size effects in visual recognition memory are determined by perceived size. Memory \& Cognition, 20, 83-95.

Nelson, D. L., Reed, V. S., \& Walling, J. R. (1976). Pictorial superiority effect. Journal of Experimental Psychology: Human Learning \& Memory, 2, 523-528.

PaIVIO, A. (1971). Imagery and verbal processes. New York: Holt, Rinehart \& Winston.

Phillips, W. A. (1983). Short-term visual memory. Philosophical Transactions of the Royal Society of London: Series B, 302, 295309.

SCHOOLER, J. W. \& ENGSTELER-SCHOOLER, T. Y. (1990). Verbal overshadowing of visual memories: Some things are better left unsaid. Cognitive Psychology, 22, 36-71.

SHEPARD, R. N., \& METZleR, J. (1971) Mental rotation of three-dimensional objects. Science, 171, 701-703.

Stefurak, D. L., \& Boynton, R. M. (1986). Independence of memory for categorically different colors and shapes. Perception \& Psychophysics, 39, 164-174.

(Manuscript received February 5, 1993; revision accepted for publication February 21, 1994.) 